\title{
Direct CW-Laser Writing Sub-Diffraction-Limit Nanopore Array Based on the Low One-Photon Absorption of Polymer
}

\author{
Fan Lianbin $^{1,2}$, Zhang Chen ${ }^{1,2}$, Li Hongfu ${ }^{1,2,3}$, Wang Kaige ${ }^{1,2}$, Feng Xiaoqiang ${ }^{1,2}$, \\ Zhao Wei ${ }^{1,2}$, Wang Weichao ${ }^{1,2}$, Bai Jintao ${ }^{1,2}$ \\ ${ }^{1}$ State Key Laboratory of Cultivation Base for Photoelectric Technology and Functional Materials, Laboratory of Optoelectronic Technology of \\ Shaanxi Province, National Center for International Research of Photoelectric Technology \& Nano-functional Materials and Application, Xi'an \\ 710069, China; ${ }^{2}$ Northwest University, Xi'an 710069, China; ${ }^{3}$ Kunming Institute of Physics, Kunming 650223, China
}

\begin{abstract}
A large area nanopore array with controllable consistency has been fabricated by direct laser writing (DLW) technique with ultralow one-photon absorption (LOPA). In this technique, a doughnut-shape beam generated by $532 \mathrm{~nm}$ continuous-wave laser through a vortex phase plate was focused into the thin film of SU8 photoresist by a high numerical aperture objective. The low absorption of the photoresist at the excitation wavelength allowed controllable fabricating structures with sub-diffraction-limit feature size and high aspect ratio. With point-by-point scanning fabrication, nanopore array with the pore's internal diameter, about ten percent of incident laser wavelength, far smaller than Abbe's diffraction limit was achieved. The influences of the exposure intensity and exposure time on the fabricated nanopore size and shape were also investigated by scanning electron microscope (SEM).
\end{abstract}

Key words: nanopore; direct laser writing; ultralow one-photon absorption; doughnut-shaped beam

Nowadays, nanopore array is one of the most indispensable functional structures in the modern scientific studies and practical applications. It has been suggested as vitally valuable structures in many advanced fields such as solar cell industry ${ }^{[1]}$, gas detection ${ }^{[2]}$, the manipulation and detection of biomolecules ${ }^{[3-5]}$, micro-/nanoscale fluidics, the lab-on-a-chip technology ${ }^{[6]}$, and especially in DNA molecules sequencing ${ }^{[7]}$. With the dramatic demand increasing of the nanopore array, many nanoscale fabricating methods and technologies have been developed for obtaining nanopores with smaller internal diameter and better consistence.

Due to the advantages of fabricating speediness, maskless process, controllable pattern shape and size, and capability of lithography in three dimension (3D), direct laser writing (DLW) has been considered to be one of the most convenient approaches in MEMS (Micro Electro Mechanical System) fabrication ${ }^{[8-10]}$. The break-through of lithographing sub-diffraction-limit feature size $^{[11]}$ makes laser writing competitive to other direct molding methods (chemical vapor deposition, focused ion beam and electronic beam lithography, nanoimprint, etc. ${ }^{[12-15]}$ in precise nanoscale structures array preparing. Ya Cheng's group has fabricated nanostructures with sub-50 nm feature size by femtosecond laser writing lithography ${ }^{[16]}$. Min Gu has shown a single silver dot at $22 \mathrm{~nm}$ fabricated by direct laser writing through highly sensitive two-photon photoreduction ${ }^{[17]}$. In Fischer's work, the nanolines with a $20 \mathrm{~nm}$ lateral feature size were written by photo-initiation inhibiting technology ${ }^{[18]}$. Jingsong Wei has achieved the lithographic feature size down to $54.6 \mathrm{~nm}$ by maskless direct laser writing nanolithography as a function of the combination of diffractive optical element and the nonlinear absorption inorganic resists ${ }^{[19]}$. Rajesh Menon prepared lines as small as $70 \mathrm{~nm}$ by optical-super-resolution nanolithography ${ }^{[20]}$. Lai's research group has obtained the 2D with a feature size of about $57 \mathrm{~nm}$ by direct laser writing technique induced one-photon absorption in a positive photoresist $^{[21]}$. Wang's group has fabricated the nanopillar array

Received date: January 08, 2017

Foundation item: National Natural Science Foundation of China (11504294, 61378083, 91123030); Natural Science Foundation of Shaanxi Province (2016JQ1030, 14JS106, 2013SZS03-Z01)

Corresponding author: Wang Kaige, Ph. D., Professor, Institute of Photonics \& Photon Technology, Northwest University, Xi'an 710069, P. R. China, E-mail: wangkg@nwu.edu.cn 
with $48 \mathrm{~nm}$ diameter by continuous optical phase modulation technology ${ }^{[22]}$. Jaroslaw Jacak, et al. have achieved the surface reative nanostructures with a feature size of $\lambda / 11$ fabricated by the stimulated emission depletion (STED) lithography ${ }^{[23]}$.

Two excitation ways namely. OPA (one-photon absorption) and TPA (two-photon absorption) have been carried out for photo-induced fabrication of sub-micrometer structures. The $\mathrm{TPA}^{[24]}$ realizes substantial three-dimensions scanning within the photoresist for its local nonlinear absorption effect. In this case, a femosecond laser at a center wavelength about $800 \mathrm{~nm}$ is applied to complete the process, and the polymer resin selected are fully transparent. This method, on the other hand, requires expensive laser sources and optical components. Recently, however a continuous-wave visible laser has been employed to fabricate all-dimensional structures based on a kind of OPA method $^{[25]}$ by matching up photoresists with an ultralow absorption band at the employed laser wavelength, which almost achieve the same results created by TPA method. In this case, the photoresist is used to possess a very smaller OPA cross-section at the laser wavelength, so that the laser beam could penetrate deeply through inside the material. Subsequently, a high NA objective tightly converge the light to the focal spot that the intensity is increased by about $10^{7}[26]$ folds compared with that at the incident beam due to the propagation of laser beam towards the focusing region following a nonlinear wave equation for the electromagnetic field. Inside the material, the physical, chemical or mechanical properties changes, are highly dominant at the focus region. This direct laser writing based on ultralow one-photon absorption (LOPA) is combined with the advantages of the OPA and TPA fabrication techniques. It is also important to note that the feature size of structures fabricated by the LOPA-based DLW technique ${ }^{[27]}$ is limited to hundreds of nanometers thanks to the diffraction limit effect at the focusing spot of a high NA microscope.

In this research, we report nanopore array with a pore diameter far smaller than Abbe's diffraction limitation by visible continuous-wave (CW) LOPA-based DLW. A $532 \mathrm{~nm} \mathrm{CW}$ laser beam, which is modified by a vortex phase mask to generate structured focus with a nanoscale dark core in the focal plane, is proposed and applied in the fabrication of the nanopore/pillar. Hence, large area consistency controlled nanopore array are attained by employing the laser beam combined with a negative tone photoresist, while nanopillar arrays will be generated with a positive tone photoresist. To the best of our knowledge, the nanopore array can be fabricated with the special properties and functions by structured visible $\mathrm{CW}$ laser. We then demonstrate experimentally that the fabrication of nanopore array with controllable and consistency pattern is possible by modulating the exposure time and exposure power.

The experimental results are measured by scanning electron microscopy (SEM). The influence of exposure doses on the inner diameter and the morphology of nanopores are finally discussed in detail. The topography and structure of the nanopores matches with the numerical simulation of the light intensity distribution. By exploiting an ultra low one-photonabsorption threshold character of the photon resist, the size changing of the nanopore is clearly analyzed.

\section{Experiment}

For our experiments, we built up a DLW system, which mainly consisted of a continuous-wave laser, a vortex phase plate (PP), and a high numerical aperture objective lens. The schematic to fabricate nanopore structure is shown in Fig.1a, 1b and 1d. The experimental setup is presented in Fig.1e. The laser source was a continuous-wave $(\mathrm{CW})$ green laser operating at a center wavelength of $532 \mathrm{~nm}$ (Verdi-V6, CONERENT, 5100 PATRICK HENRY DR. SANTA CL. CA 95054). A spatial filter was placed behind the laser bead to attain a high-quality beam mode. A $\lambda / 2$ wave plate (WP) was used to modulate polarization direction of laser beam. The laser beam then propagated via a $\lambda / 4$ wave plate (WP) to achieve a well right-handed circularly polarized beam. The PP in anticolockwise direction was used to generate a doughnut beam with a 0 intensity at the center. A objective lens with high numerical aperical (NA=0.9, Car Zeiss MicroImaging GmbH, Jena, Germany) was employed to focus the beam. The dimension of the center dark core was far smaller than that of the diffraction limit. Therefore, such high NA microscope allowed to elaborate a structure with a submicrometer resolution through a thin film coated on glass substrate. During experiments, the photoresist-coated glass substrate was transferred onto a three-dimensional (3D) piezo-electric scanning stage (M-521.DG NanoCube, Physik Instrutmente PI, Karlsruhe, Germany) to hold and move samples. We used a PC program to rapidly drive the motion of PI stage. A mechanical switch reaching a time resolution about $10 \mathrm{~ms}$ was used to control laser path. A high-performance digital charge-coupled device (CCD) camera was used to monitor the back-reflected beams from the sample surface transmitting a Dichroic Mirror (DM) before the objective lens in order to adjust dimension of the focus at the focal plane, thus determining whether a focusing spot, locates on the focal plane by its imaging. Fig. $1 b$ shows the laser spot imaged in the focal plane by the $\mathrm{CCD}$, i.e. the doughnut-shaped pattern on the focal plane. However, this pattern of laser beam was used to create a nanopore structure with feature size below diffraction limit shown in Fig.3a.

The photoresist used in this work was a commercial SU8 negative-tone photoresisit, with a ultra-low absorption in the visible range, as shown in Fig.1c, in particular at $532 \mathrm{~nm}$ wavelength which is located at the red edge of the absorption band, and the absorption was very low and likely to close to 0 . Although the absorption was ultra low, the effective excitation can be achieved at tightly focusing spot, where the excitation intensity was sufficient to compensate the absorption of the SU8 photoresist $^{[28]}$. As a consequence, a kind of controllable 

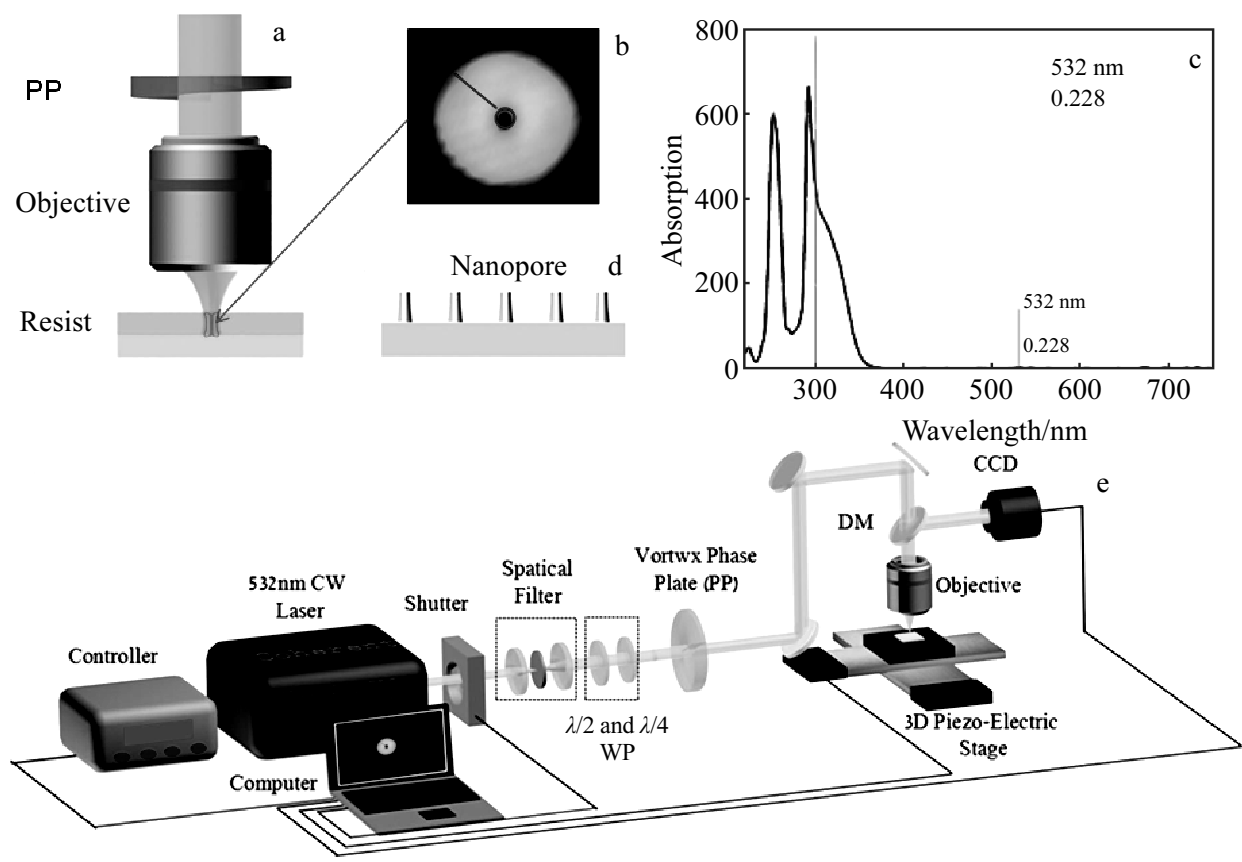

Fig.1 Schematic experimental set up for LOPA-based DLW technique with a vortex phase plate and a continuous-wave $532 \mathrm{~nm}$ beam: (a) illustration of the creation of desired structures on a SU8 photoresist, (b) the focusing spot located inside material, (c) the absorption spectrum of SU8 photoresist at continuous-wave $532 \mathrm{~nm}$, (d) the nanopore structure created on the glass substrate, and (e) the direct laser writing system to fabricate nanopore arrays

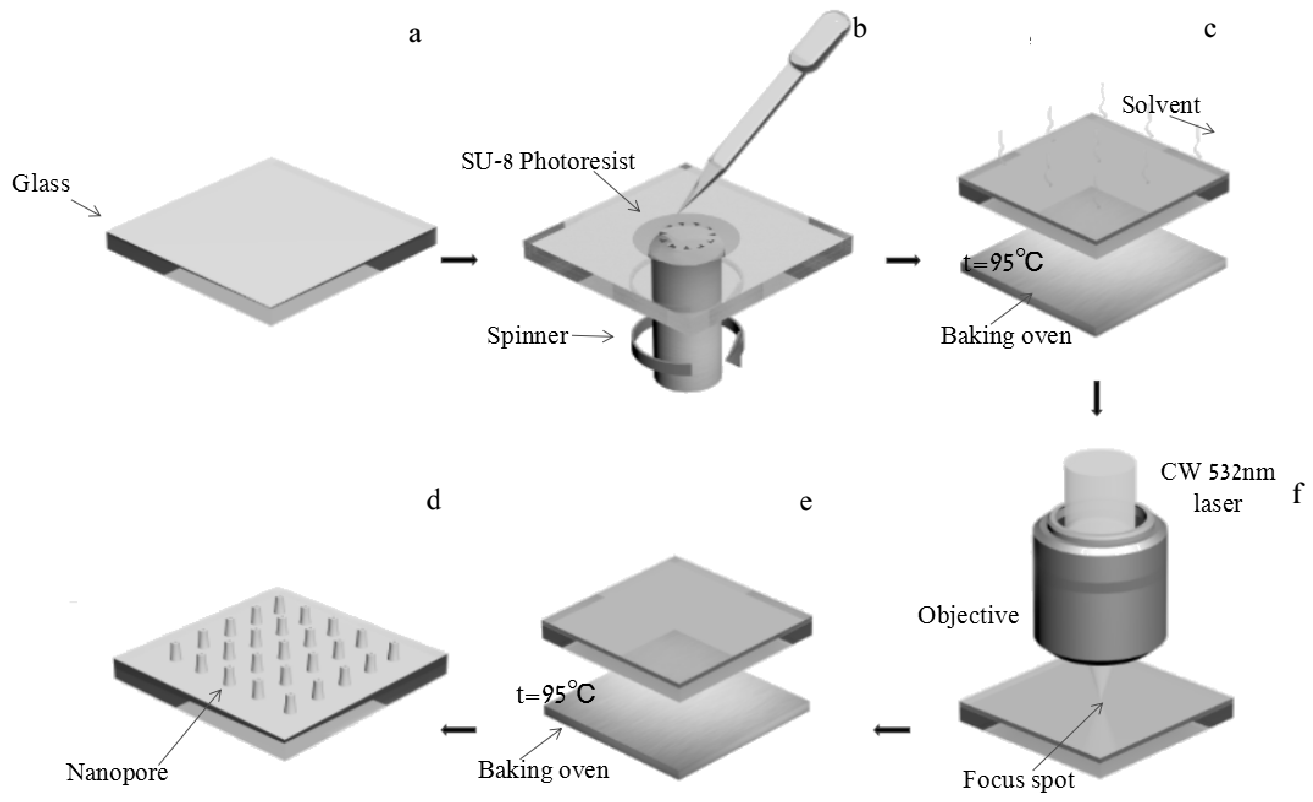

Fig.2 Illustration of the fabrication process of large area controllable consistency nanopore array: (a) glass substrate preparation, (b) SU8 photoresist spin-coated on the performed glass substrate, (c) prebake stage to evaporate solvent on the Baking oven at $95{ }^{\circ} \mathrm{C}$ for $20 \mathrm{~min}$, (d) the SU8 photoresist was exposed by using a direct laser writing system with a continuous-wave $532 \mathrm{~nm}$ laser, (e) postbake stage to induce the cross-linking on the Baking oven (e) at $95^{\circ} \mathrm{C}$ for $20 \mathrm{~min}$, and (f) the nanopore can be obtained by developing process

nanostructures can be fabricated by the LOPA-based DLW through modulating exposure power or exposure time. Fig. 2 is the fabrication procedure of the nanopore array. To fabricate nanopore structures, the SU8 photoresist was firstly spin-coated on immaculate glass substrates, which was then placed in a clean environment for about $24 \mathrm{~h}$ to flatten sample surface, and next pre-baked for $2 \mathrm{~min}$ at $65{ }^{\circ} \mathrm{C}$ and $20 \mathrm{~min}$ at $95{ }^{\circ} \mathrm{C}$ on a Baking oven to remove the residual solvent. The exciting laser beam can be tightly confined within a hundred nanometer spot by an high NA microscope. When a single exposure and a 

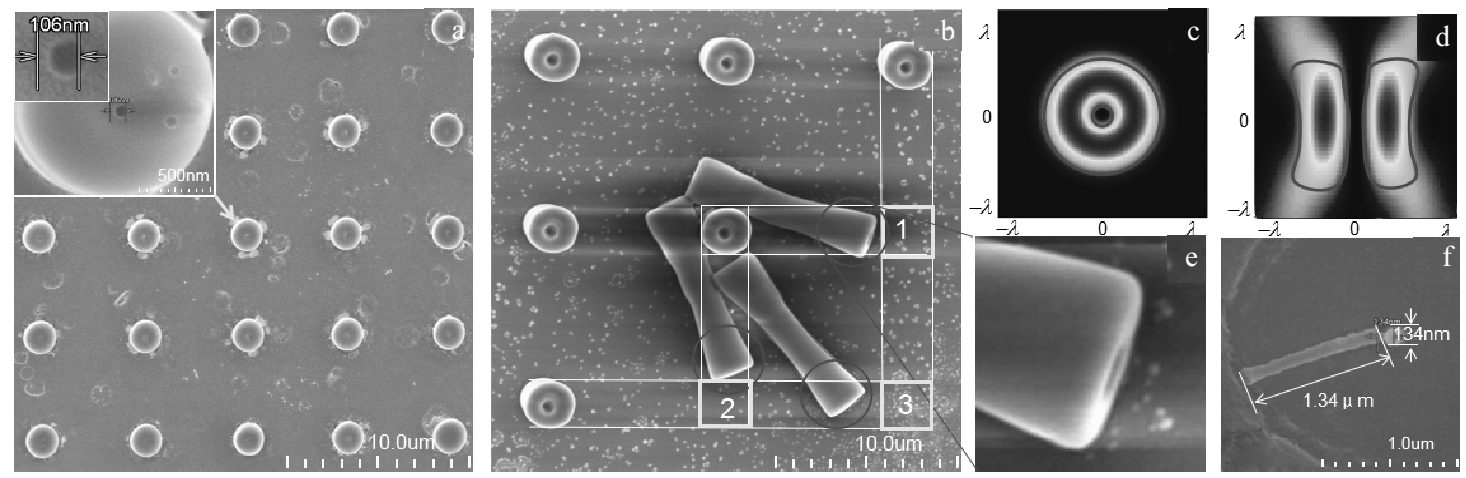

Fig.3 (a) SEM image of nanopore array, for which the voxels with the uniform structure morphology and feature size, the chart is one of the nanopore array with the feature size of $106 \mathrm{~nm}$; (b) SEM image of the morphology of nanopore structure at the $x-y$ plane (upright pillars) and the $x-z$ plane (dumping pillars); (c, d) The intensity distribution of the numerical simulation about dount-shaped focal spot at the $x-y$ plane and the $x-z$ plane, respectively. The region surrounded by the red curve can be induced the effective photopolymerization to create a solid voxel; (e) The partial enlarged detail of the bottom of nanopore voxel; (f) SEM image of a dumpling nanopillar, which was fabricated by the lithography system

post-bake process were made, a single voxel corresponding to a volume in which the exposure dose was above the polymerization threshold can be obtained. And the exposed process generated a strong acid due to the absorption of one or multiple photons. After exposure, the samples were placed in baking oven to posting-bake at $95{ }^{\circ} \mathrm{C}$ for $20 \mathrm{~min}$ which is a major step to induce the cross-linking. To obtain nanopore structure standing in the glass substrate need to bath the sample in SU8 developer for about $60 \mathrm{~s}$ and then rinse residue attached to the surface with isopropyl-ketone. All unexposed parts were removed resulting in desired structures. The obtained nanostructures were measured, characterized, and analyzed with a scanning electron microscope (SEM, SU8010, HITACHI).

\section{Results and Discussion}

By a standard fabrication process, we demonstrate that a nanopore structure is fabricated only at the inner part of the focusing region by controlling the exposure doses and structures periods; the $5 \times 5$ nanopore array structure, for example, has been fabricated by scanning the focusing spot along $x-y$ plane. By precisely setting the laser power and the exposure time, nanopore structure with a feature size was created. Fig.3a shows a typical SEM image of $2 \mathrm{D} 5 \times 5$ nanopore array structure with $\Lambda=25 \mu \mathrm{m}$ fabricated with a optimized laser power and an established exposure time by the LOPA-based DLW technique. We note that every nanopore voxel attached on the glass substrate possesses identical structure morphology and feature size. Furthermore, the nanopore structure with a pore diameter of $106 \mathrm{~nm}$ is far smaller than the size of Abbe's diffraction limit with half of the wavelength used. Fig.3b particularly exhibits the SEM image of dumping nanopore voxels which are provided with the same lateral morphology and corresponded to the energy distribution of focusing spot intensity at $x-z$ plane. In addition, we observed that the structure fabricated have high aspect ratio and consistency.
The dumping nanopore voxel should be located on the position of yellow boxes, according to the position of the intersection of the four white lines along the vertical direction and horizontal direction, which demonstrates that the region of red circle belongs to the bottom of nanopore structure. Fig. $3 \mathrm{c}$ and $3 \mathrm{~d}$ are the results of the numerical simulation, which is based on diffraction integration theory, and calculated by MATLAB program, to show the intensity distribution and the pattern of the focal spot on the $x-y$ plane and the $x-z$ plane, respectively. The red curve represents the effective region to induce complete photopolymerization, in which exposure dose is above the polymerization threshold. As a consequence, a desired voxel can be obtained by LOPA-based DLW, shown in Fig.3b. Fig.3e shows the bottom structure with respect to the nanopore voxel, and we can clearly notice that there is an identical pore at the bottom position, yet. It reveals that no or a few photons have radiated to the region to induce polymerization, in which SU8 photoresist can be washed out in developing process. Fig.3f exhibits a slender fall nanopillar experimentally fabricated with the width of about $134 \mathrm{~nm}$ and the length of approximately $1.34 \mu \mathrm{m}$ by the same laser focusing spot (see Fig.1b) and a positive photoresist for continuous-wave 532 $\mathrm{nm}$ laser. It is worth noting that the homogeneous width and the high aspect of the nanopillar illustrate that there exists an approximate cylindrical channel located in the center of the focusing spot. Hence, we believe that when the laser beam is focused into the negative photoresist thin film, there should be no or only a few photons which can reach the region to induce photochemical reaction. Meanwhile, the nanopore of the high aspect ratio and the similar homogeneous width can be experimentally fabricated with the SU8 photoresist and the CW $532 \mathrm{~nm}$ laser by the LOPA-based DLW. Therefore, the channel inside the cylindric microstructure should be smooth and open with an appropriate exposure power and time. From Fig.4, it is obvious that the LOPA polymerization process emerges in the 

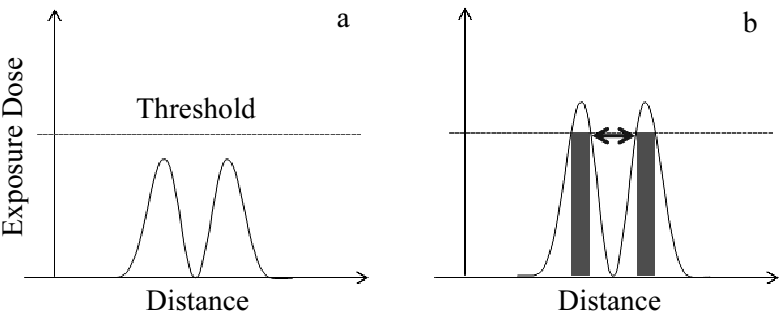

Distance
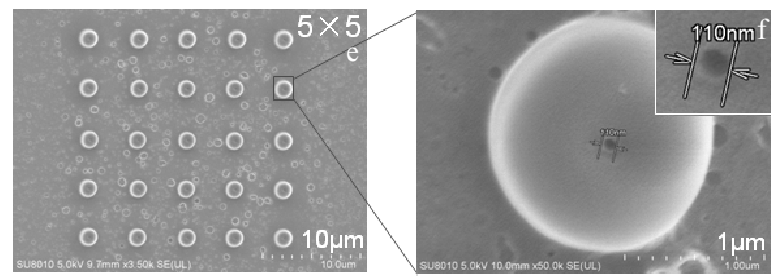

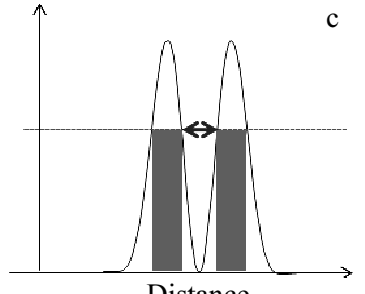

Distance

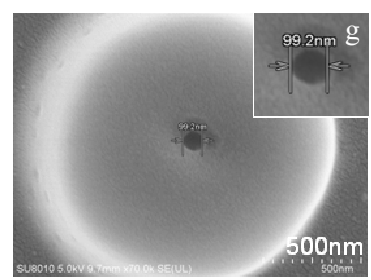

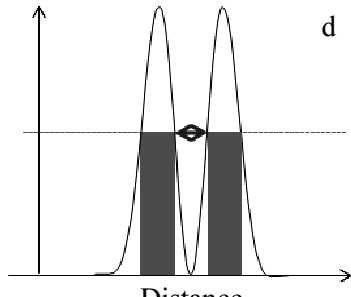

Distance

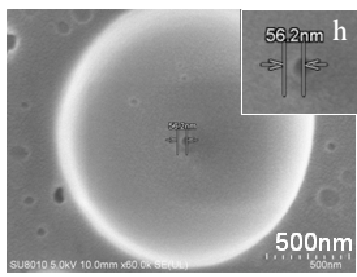

Fig.4 Schematic representation of lateral localization in microscopy (a d) and SEM images of nanopore structure (e h): (a) the intensity distribution of the focusing spot at $x-y$ plane, the red dotted line represents the fully polymerization threshold of the SU8 photoresist, the bimodal curve indicate the exposure dose, DLW with polymerization threshold pushed to the peak of the illumination PSF. In an area where the PSF is above the polymerization threshold are solidified sufficiently to withstand development (green area); (b) (c) and (d) the green part symbolize the region induced fully photopolymerization, and the blue double-headed arrow lines indicate the distance $(\Delta r)$ of nanopore. (e) SEM image of $5 \times 5$ nanopore structure array; (f), (g) and (h) SEM images of a single nanopore structure with different feature sizes

area of the focusing spot where the excitation intensity is very high. A complete nanopore channel can be fabricated by modulating laser power and exposure time to an appropriate value. The top topographic image is similar to the focusing spot shown in Fig.1b. First, we have fabricated nanopore structure on glass substrate with applicable exposure dose for ultralow one-photon absorption (LOPA) lithographing. The minimal nanopore feature size is found at laser power of about $60 \mu \mathrm{W}$ and an exposure just lasting $800 \mathrm{~ms}$. This allows the fabrication of nanopore with a diameter of $56.2 \mathrm{~nm}$ (see Fig.4h). Apparently, this direct laser writing based on LOPA can be employed to create the micro/nanostructures of the controllable feature size and shape by modulating exposure dose. It is noted that, in the case of LOPA, there exists a fixed exposure dose threshold, above which the polymerization process can be fully completed. In addition, due to a high intensity of the focal spot, the complete photo-polymerization only happens in this region. Therefore, the 2D and 3D structures could be obtained by continuous-wave laser. As is shown in Fig.4e, a typical $5 \times 5$ nanopore array was experimentally fabricated. Each nanopore voxel stands upright on the glass substrate orderly and the adjacent voxels are separated by $25 \mu \mathrm{m}$ with periodic arrangement. The average inner diameter size of the nanopore is 62.5 $\mathrm{nm}$. It is much smaller than the size of Abbe's diffraction limit. Fig. $4 \mathrm{f}, 4 \mathrm{~g}$ and $4 \mathrm{~h}$ are the results with different exposure dose. It is seen that the inner diameter feature sizes of nanopore with different exposure dose are different. The evolution of nanopore's size and shape fully illustrates the feature size is controllable in the LOPA process. Furthermore, Fig.3a, 3b, 3c and $3 \mathrm{~d}$ reflect the relationship of the polymerization threshold of SU8 photoresist and the exposure dose for the nanopore feature sizes. Because the polymerization threshold is a constant on exposure dose, the photopolymerization process can be fully completed to create a solid hollow structure. In fact, the light-material interaction region is confined to the small volume within the focus of the laser beam. The diameter of the nanopore beyond the diffraction limit can be realized by controlling exposure dose. With careful calibration and analysis, the nanopore feature size has reached a minimal value of 56.2 $\mathrm{nm}$, which is around $\lambda / 10$, much smaller than the diffraction limitation of $\lambda / 2$, where $\lambda$ is the incident laser wavelength. Generally, the feature size of the nanopore is governed majorly by the size focal spot, which is about $0.61 \lambda / \mathrm{NA}$. It is possible to realize structures with very small size by controlling the exposure dose precisely. We can see that the nanopore feature sizes could be gradually reduced by following the increase of the exposure dose from $110 \mathrm{~nm}$ to $56.2 \mathrm{~nm}$. We believe that it is possible to obtain nanopore structure with feature size below 50 $\mathrm{nm}$ by microscope with higher NA.

Fig. 5 shows SEM images of a $5 \times 5$ nanopore array and the comparison between the random collected nanopore structures from the array. It is noted that the nanopore structures, which are located at different positions of glass substrate, possess high consistency of morphology. The morphology of a single nanopore structure depends on the shape of the laser focal spot which fully induces photopolymerization of the photon resist. While the feature sizes depend on the exposure dose of the laser. In order to fabricate the large area controllable nanopore array possessing high consistency by LOPA-based DLW, we can make a large area scanning in $x-y$ plane following a pre- 


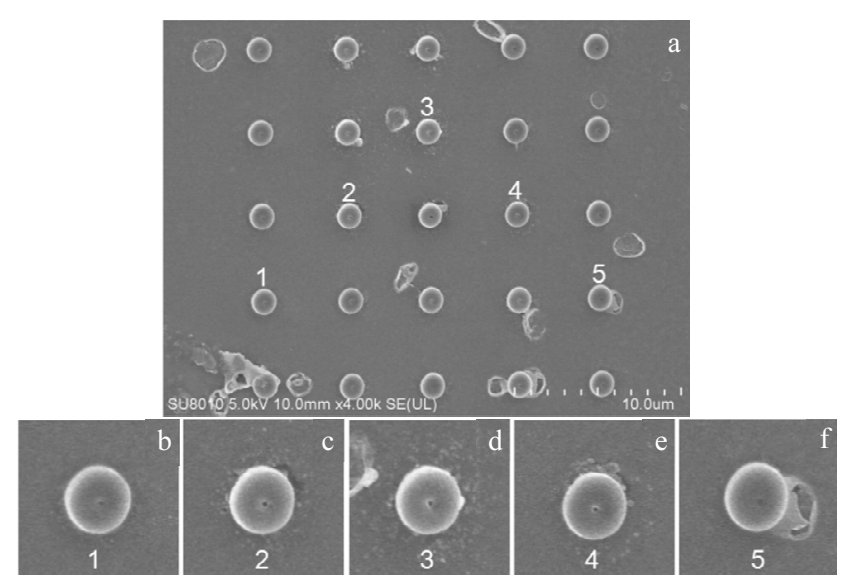

Fig.5 SEM images of $5 \times 5$ nanopore array and single nanopore structure: (a) the large area controlled consistency nanopore array, and $(b \sim f)$ represent the morphologies located on 1 to 5 position, respectively

configured lattice coordinates with the same exposure dose.

\section{Conclusions}

In this study, a direct laser writing technique with doughnut-shape beam is developed by $532 \mathrm{~nm}$ continuous-wave laser and far-field super-resolution microscopy system. Under the low operation power and corresponding ultra-low absorption of SU8 photoresist, a nanopore array with $56.2 \mathrm{~nm}$ core diameter has been realized. The core diameter is only $1 / 10$ of the $532 \mathrm{~nm}$ excitation beam. Furthermore, we find the diameter of nanopore has approximately linear relation with exposure time. This is especially helpful on applying the ultra-low OPA direct laser writing technique in practice.

Our work has created a novel nanopore array. 2D/3D nanostructures can be fabricated with comparable resolutions as TPA direct laser writing techniques. This research verifies the feasibility of using the ultra-low OPA direct laser writing technique to fabricate nanostructures at all dimension in the future. Meanwhile, a continuous-wave laser instead of a mode-locked femtosecond laser can also significantly reduce the cost, and tremendously increase the reliability as well as the stability of such advanced optical lithography systems.

\section{References}

1 Chattopadhyay S, Huang Y F, Jen Y J et al. Materials Science and Engineering: R: Reports[J], 2010, 69(1): 1

2 Ten Bosch A. Separation and Purification Technology[J], 2006, 47(3): 156
3 Kasianowicz J J, Brandin E, Branton D et al. Proceedings of the National Academy of Sciences[J], 1996, 93(24): 13770

4 Branton D, Deamer D W, Marziali A et al. Nature Biotechnology [J], 2008, 26(10): 1146

5 Venkatesan B M, Bashir R. Nature Nanotechnology[J], 2011, 6(10): 615

6 Yuqing M, Jianrong C, Keming F. Journal of Biochemical and Biophysical Methods[J], 2005, 63(1): 1

7 Venkatesan B M, Bashir R. Nanopores[M]. New York: Springer, 2011: 1

8 Sekkat Z, Kawata S. Laser \& Photonics Reviews[J], 2014, 8(1): 1

9 Hohmann J K, Renner M, Waller E H et al. Advanced Optical Materials[J], 2015, 3(11): 1488

10 Rebeiz G M. RF MEMS Theory, Design, and Technology[M]. New York: John Wiley \& Sons, 2004

11 Kawata S, Sun H B, Tanaka T et al. Nature[J], 2001, 412(6848): 697

12 Reina A, Jia X, Ho J et al. Nano Letters[J], 2008, 9(1): 30

13 Giannuzzi L A, Stevie F A. Micron[J], 1999, 30(3): 197

14 Vieu C, Carcenac F, Pepin A et al. Applied Surface Science[J], 2000, 164(1): 111

15 Chou S Y, Krauss P R, Renstrom P J. Journal of Vacuum Science \& Technology B[J], 1996, 14(6): 4129

16 Liao Y, Shen Y, Qiao L et al. Optics Letters[J], 2013, 38(2): 187

17 Cao Y, Gu M. Applied Physics Letters[J], 2013, 103(21): 213104

18 Fischer J, Wegener M. Laser \& Photonics Reviews[J], 2013, 7(1): 22

19 Zha Y, Wei J, Gan F. Optics Communications[J], 2013, 304: 49

20 Majumder A, Masid F, Pollock B et al. Optics Express[J], 2015, 23(9): 12244

21 Tong Q C, Nguyen D T T, Do M T et al. Applied Physics Letters [J], 2016, 108(18): 183104

22 Chen Zhang, Kaige Wang, Jintao Bai et al. Nanoscale Research Letters[J], 2013, 8(1): 1

23 Buchegger B, Kreutzer J, Plochberger B et al. ACS Nano[J], 2016, 10(2): 1954

24 He G S, Bhawalkar J D, Zhao C F et al. Applied Physics Letters [J], 1995, 67(17): 2433

25 Bhat R D R, Nastos F, Najmaie A et al. Physical Review Letters [J], 2005, 94(9): 096603

26 Tong Q C, Do M T, Journet B et al. International Society for Optics and Photonics [C]. Bellingham WA: SPIE Photonics Europe, 2016: 988519

27 Do M T, Nguyen T T N, Li Q et al. Optics Express[J], 2013, 21(18): 20964

28 Nguyen D T T, Tong Q C, Ledoux-Rak I et al. Journal of Applied Physics[J], 2016, 119(1): 01 


\title{
基于聚合物低单光子吸收连续激光直写技术研制亚衍射极限纳米孔阵列
}

\author{
范连斌 1,2 , 张 琛 $^{1,2}$, 李红福 ${ }^{1,2,3}$, 王凯歌 ${ }^{1,2}$, 冯晓强 ${ }^{1,2}$, 赵 伟 $^{1,2}$, 王伟超 ${ }^{1,2}$, 白晋涛 ${ }^{1,2}$ \\ (1. 陕西省光电子技术与功能材料重点实验室, 陕西省光电子技术重点实验室, \\ 国家级光电子技术与功能材料及应用国际科技基地, 陕西 西安 710069) \\ (2. 西北大学, 陕西 西安 710069) \\ (3. 昆明物理研究所, 云南 昆明 650223)
}

\begin{abstract}
摘 要: 基于单光子吸收激光直写技术, 成功地制备了大面积阵列分布、一致性可控的纳米孔复合结构。通过一种浴旋相位板的调制, $532 \mathrm{~nm}$ 波段的连续激光在高数值孔径物镜的焦点处形成了一个圆环形光斑, 作用于均匀的 SU8 光刻胶薄膜上, 进而制备出形状与大小 可控的、具有高深宽比、直径突破阿贝衍射极限的纳米孔结构。通过逐点扫描方法, 最终制备出内径仅为入射波长约 $1 / 10$ 的纳米孔阵 列结构。此外, 利用 SEM 技术研究了曝光光强和曝光时间对纳米孔尺寸和形貌的影响。
\end{abstract}

关键词: 纳米孔; 激光直写; 超低单光子吸收; 环形光束

作者简介: 范连斌, 男, 1989 年生, 硕士生, 西北大学光子学与光子技术研究所, 陕西 西安 710069, E-mail: 2356021208@qq.com 